\section{Medical Care in the Arctic and on Orbit}

David Saint-Jacques*

I joined the Canadian Space Program in 2009. I am currently undergoing basic astronaut training leading, hopefully for a future mission aboard the Intenational Space Station (ISS). My perspective is therefore that of a newcomer without spaceflight experience. I do, however, have some experience as my understanding of how medical care is provided on orbit grows, it has been interesting for me to see the parallels with my former practice.

MacGyver spirit

The first thing that comes to mind when to their colleagues working in large centers, northern first-line physicians are more on their own. The absence

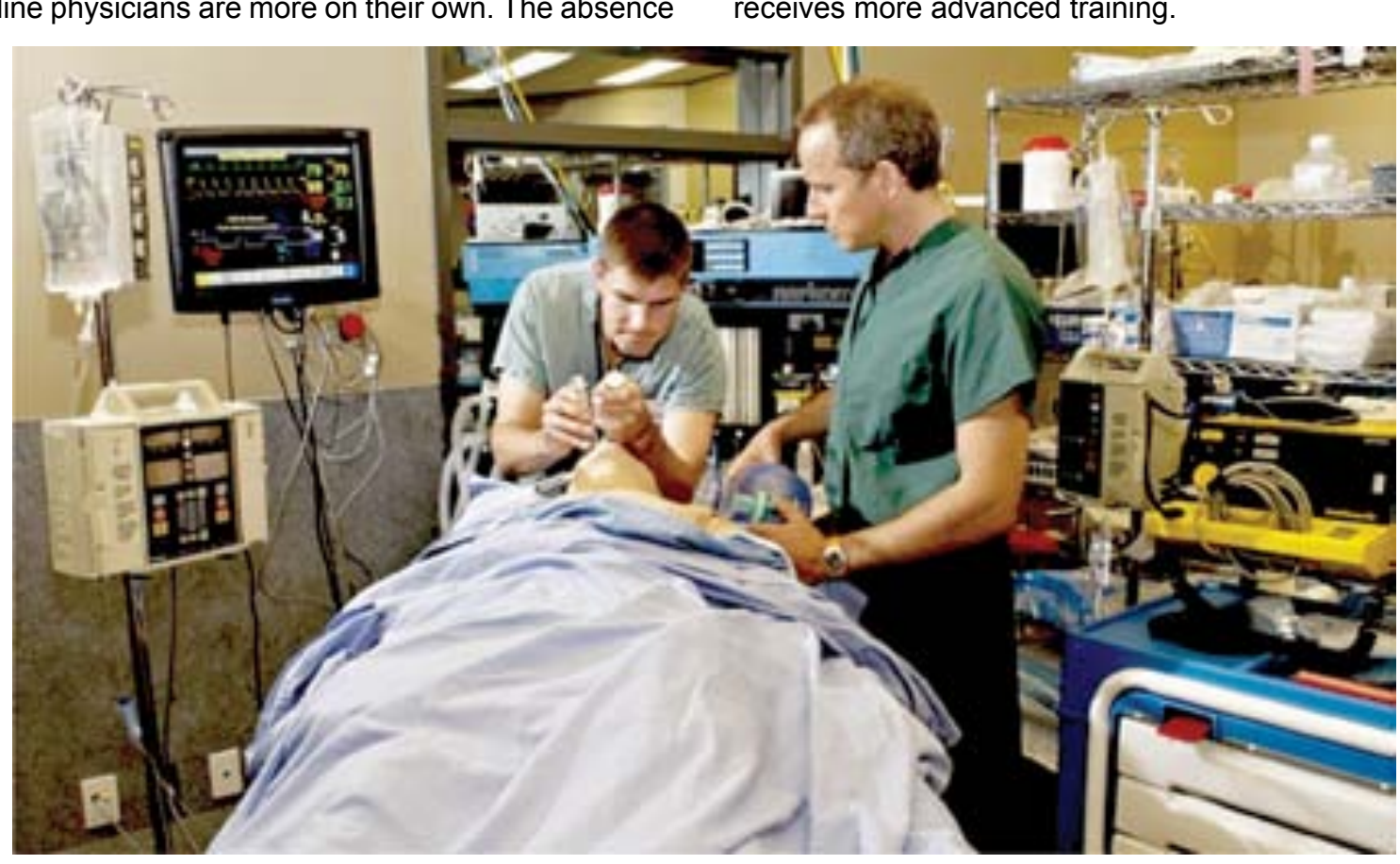

Figure 1: Intubation training of readily available consultants has a big impact on their practice; there is a strong incentive to strive for maximum autonomy and to stay current on as many topics as possible. Diagnostic tools and therapeutic

All of that is also true of the Crew Medical Officer (CMO) on a space mission. Readiness, inventiveness, outside-the box thinking and a broed knowledge base are important to all physicians, but are particularly key to those working on their own in remote locations Incidentally, there is not always a physician on-board the ISS; every crewmember receives basic

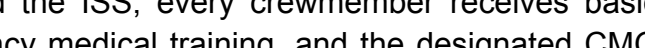
eceives more advanced training.

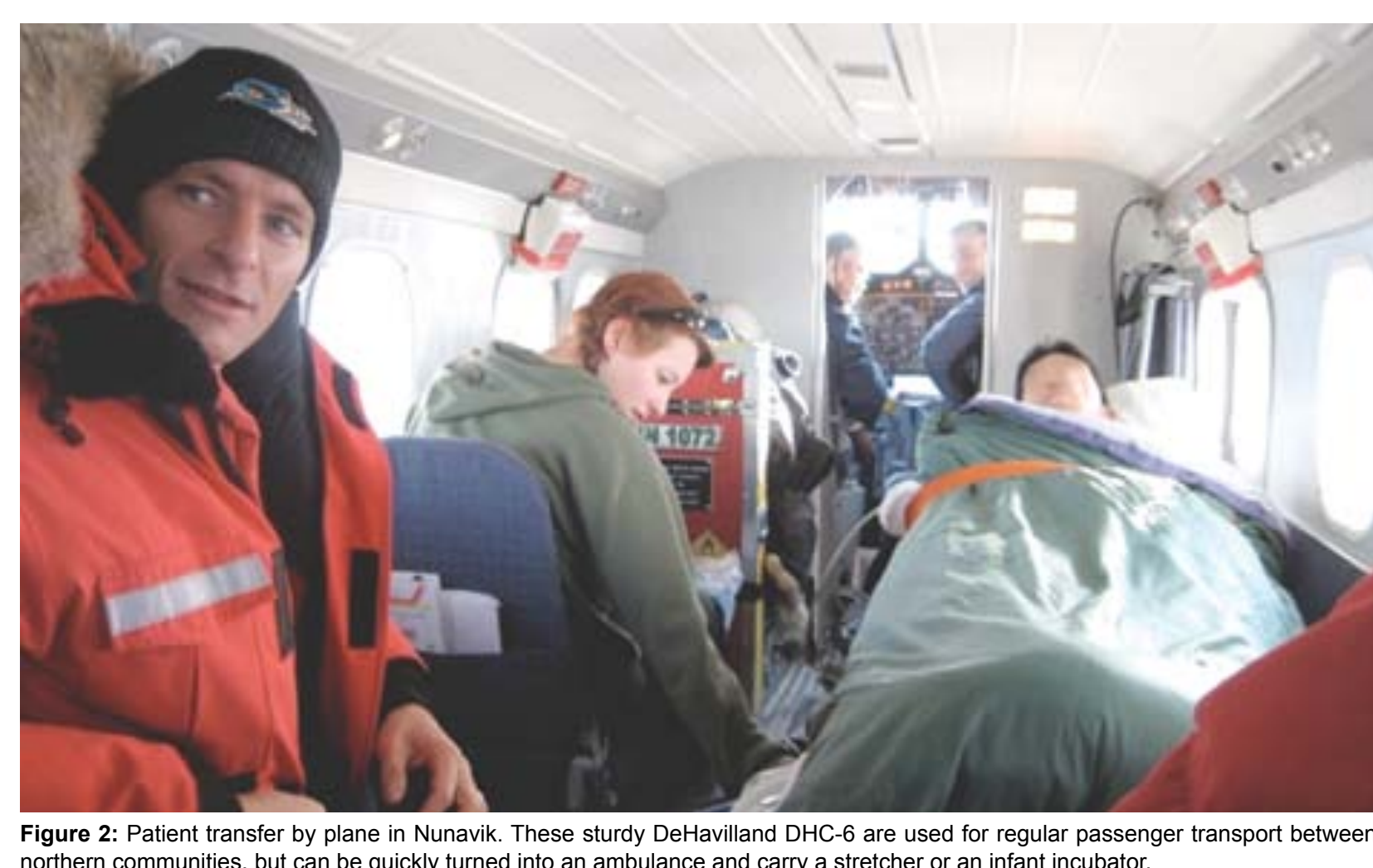

Figure 2: Patient transfer by plane in Nunavik. These sturdy DeHavilland DHC-6 are used for regular passenger
northern communities, but can be quickly turned into an ambulance and carry a stretcher or an infant incubator.

Telehealth

Of course, up north, one is not completely alone: help is always available in one form or another thanks to telehealth. For physicians working in northern communities, telehealth most often simply means a phone call to a specilist. Salethe intern thow allows the transmission of clinical photographs and locally obtained $x$-rays. Where and when videoconferencing bandwidth permits, one can even perform ultrasound under the live guidance of a remote specialist. This has, for example, revolutionized prepartum care for Inuit women, who den't have to fly "sonhe just for a regular exam. Telehealth is also a great way for specialists to provide mentoring to remole personn

All these tools currently apply to on-orbit medical care. There is always a light surgeon avallable to Earth. For example, commonly transered images include cardiovascular ultrasound loops fundosopic images and dermatological photogaphe $\mathrm{X}$-Ray MRI and CT are not available due to the prohibitive launch weight of the required equipment. Interestingly. telehealth is a good example of bidirectional technology transfer, where tools and protocols developed for terrestrial applications are used on-orbit, and viceversa. However, as we contemplate deep-space
missions back to the Moon, and on to Mars, the trade- off between support from Earth and medical autonomy becomes an issue. Basically, the further away, the more autonomy is required.

This is driven firstly by the increasing delay in communications. Since we can't transmit radio signals faster than the speed of light, from Mars it could lake up to 40 minutes to get an answer back, making consultations very cumbersome. Secondly, increasing distance makes an emergency medical evacuation less and less practical. Whereas evacuation from the lowearth orbit where ISS is located would be expensive bu possible, an evacuation from Mars would probably no

Environmental and cultural issues

There are other obvious parallels between medical practice in northern Canada and on-orbit. One is the harsh and unforgiving nature of these beautiful environments; this modifies the frequency of various pathologies and drives the requirements for preparedness. For example, up north, the occurrence of exposure is relatively high, whereas on orbit decompression accidents are a threat - fortunately, this has not happened yet!

The other parallel one can draw is the crosscultural element. Physicians working in northern Canada serve an Inuit population with different lifestyles and different expectations towards healthcare compared to 
urban Canadians. The physician must adapt to these The same is true on orbit, with crews generally international composition.

Romanow Commission: "I believe that the success of our Health Care System as a whole will be judged not by the quality or service available in the best urban facilities, but by the equality of service Canada can provide to its remote and northern communities."

Risk management

Living in a remote area is a health risk in itself for example, the chances of surviving a major hea trauma are several orders of magnitude lower when the nearest neurosurgeon and ICU are several hours away by flight. Remoteness also drives the way we organize patient follow-up for more benign ailments: norther physicians tend to err on the conservative side, general, to further minimize risks of complications.

The local population understands and accepts these risks; the challenge for northern health care providers, and the responsibility of the healthcare system, is to ensure these discrepancies are minimized,

Similar concerns apply to on-orbit medical
care. For example, in deciding the content of the onboard medical kit, one must decide what pathologies the crew could likely treat successfully. Deciding whether a particular iliness or injury is survivable or not on-orbit is a matter of ongoing speculation and debate. This uncertainty is essentially what drives the requirement for crewmembers to undergo such stringent medical screening, in the hope of minimizing risk. Again, as we envision deep-space missions wherein medical evacuation is not an option, the listically treat only gets more relevant.

David Saint-Jacques (MD, PhD) is an astronaut candidate with the Canadian Space Agency. He and is currently in basic training at NASA's Johnson Space Center in Houston, Texas. He was selected in 2009 while he was working as a family physician in the Inuti community of Puvirnituq, in Nunavik, Northern Quebec. He eceived a B.Eng in engineering physics from Ecole Polytechnique in Montreal, and worked as a biomedical engineer in Paris, France. He subsequenly oblained a PhD in astrophysics al Cambridge Unisersity, UK and Laval, and completed a residency in family medicine at McGill University.

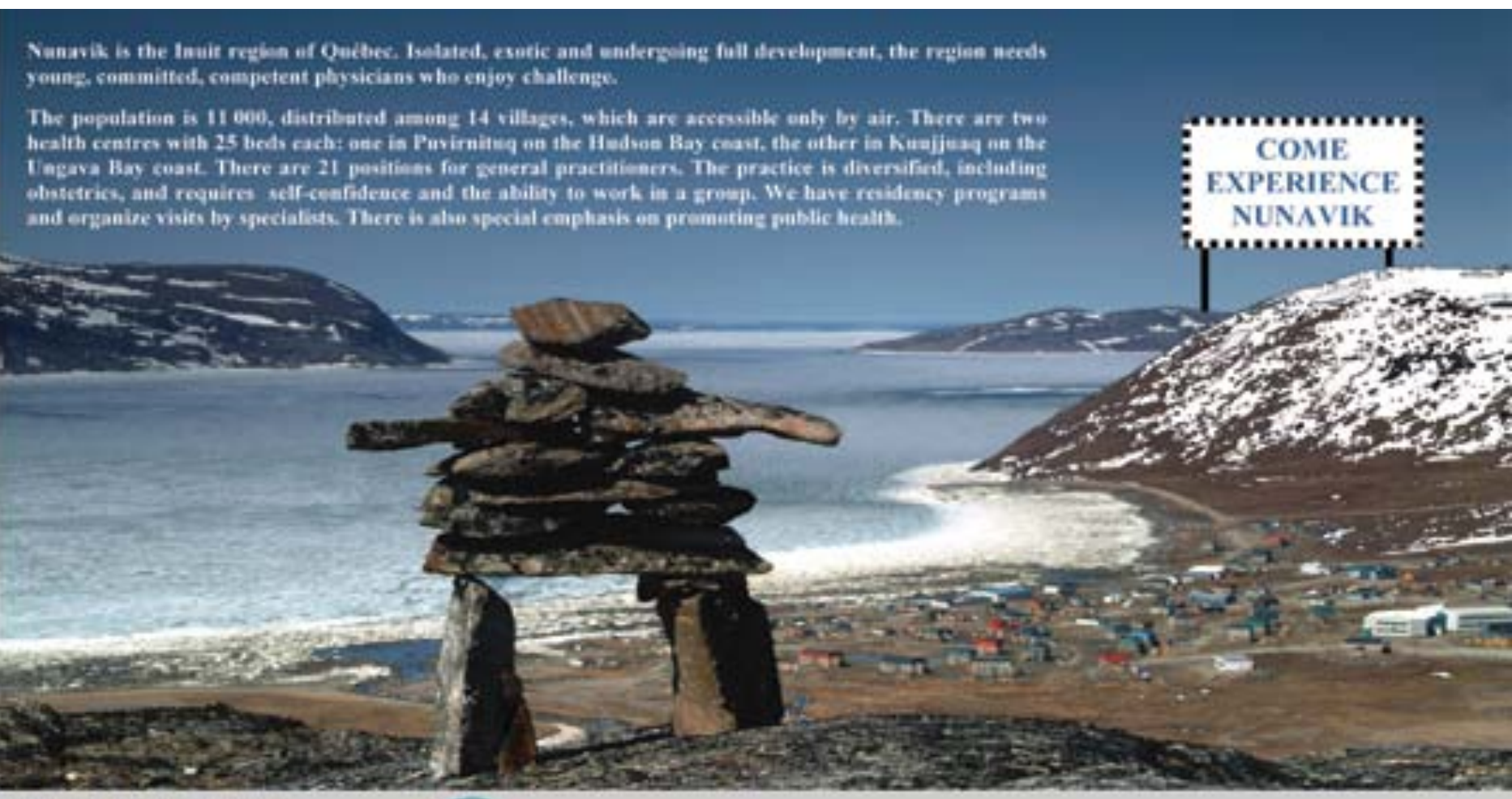

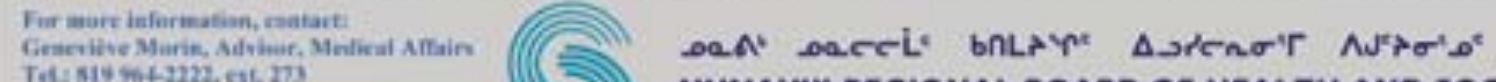

Sini sepvices REGIE RÉGIONALE DE LA SANTE ET DES SERVICES SOCIAUX NUNAVIK
CROSSROADS

\section{Physicians as Astronauts}

Robert Thirsk

In 2009 I had the good fortune to fly on a ong duration space mission. With two crewmates, I launched aboard a Russian Soyuz rocket from the Balkonur Cosmodro Russin Soyz rocket from were traveling at a speed of 28,000 kiloseters per hour through an environment devoid of air water and anything familiar. Two days later we rendezvoused with the International Space Station (ISS) at an altitude of $350 \mathrm{~km}$. As our Soyuz vehicle docked with the Station, we began an incredible space odyssey as members of the ISS Expedition 20/21 crew.

This Expedition marked the first time that the ISS hosted a permanent crew of six. My international crewmates (from Russia, the United States, Japan and Belgium) and I performed an unprecedented amount of multidisciplinary research (Figure 1). We also performed complex robotic erations, spacewalks, and work of Station systems and payloads (Figure 2). Six months later my Soyuz crewmates and I undocked from the Station and landed back in Kazakhstan. During our stay in space, we completed 3,000 orbits of the Earth and traveled 125,000,000 $\mathrm{km}$. It was truly an odyssey.

This ISS expedition as well as my earlier Space Shuttle mission have enriched me in ways I can never fully explain. I often reflect on the career some of my medical colleagues, this path seems some of my medical colleagues, this path seems of medicine have in common with space exploration?".

In the following paragraphs, I describe the astronaut profession and its commonalities with mative experience and the spaceflight environment

*To whom correspondence should be addressed.

Dr. Robert Thirsk Email: robertb thirsk@nasa gov

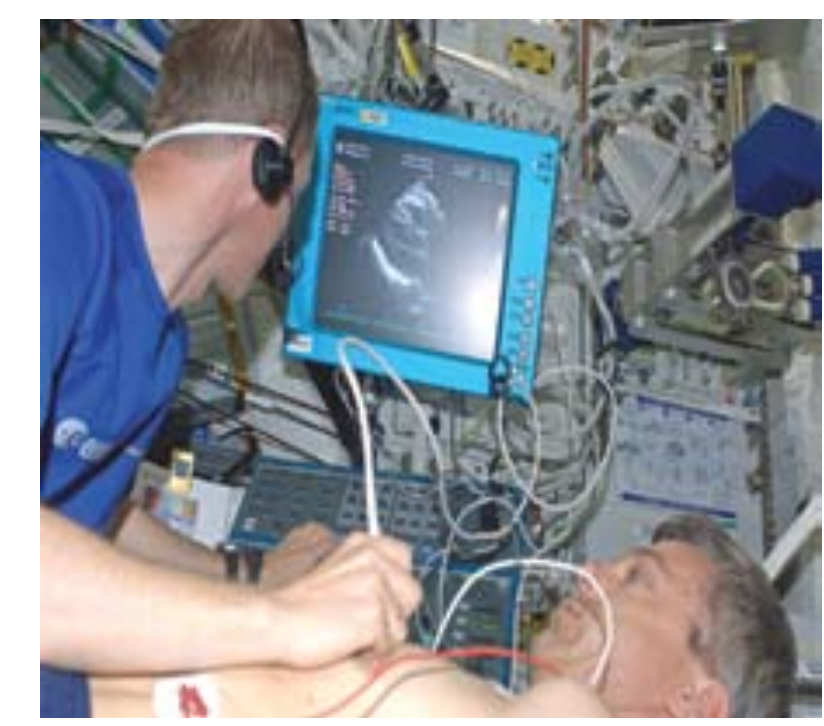

(1)

cardiography on Ro astronaut Frank De Winne performs echotigated cardiovascular adaptation to weightlessness.

is alien to anything in the clinical world. However, a career transition to space exploration after investing so much time and effort in a medical career is not unusual. A well-trained astronaut exhibits many of the same knowledge, skills and professional at tributes of an exemplary physician. Indeed, a medcal background forms an excellent foundation for
career in astronautics.

\section{SELECTION}

An astronaut career begins with selection even more protracted, competitive and rigorous than it is for medical school. The Canadian Space Agency's most recent recruitment campaign in 2008/09 lasted 12 months and saw 5,300 people apply for only two available positions.

Astronaut candidates represent a wide spectrum of professionals such as test pilots, engineers, scientists, educators and physicians. Candidates who have considerable experience working 